\title{
STRONG CONSISTENCY FOR SIMPLE LINEAR EV MODEL WITH $\psi$-MIXING ERRORS
}

\section{HU and PINGYAN CHEN}

Department of Mathematics

Jinan University

Guangzhou, 510632

P. R. China

e-mail: hudi92@foxmail.com

\begin{abstract}
In the paper, the simple linear errors-in-variables (EV) model with $\psi$-mixing errors is studied. Under suitable mixing coefficient rate and moment conditions, the convergence rates of strong consistency for the least square estimators of parameters in the simple linear EV model are obtained.
\end{abstract}

\section{Introduction and Main Result}

The regression models are widely used in many area because of their good intuitive nature and extensive applicabilities. However, the measurement errors exist during the collection and statistics data. Since the ordinary regression models do not do well in considering measurement errors, the errors-in-variables (EV) regression models come into being.

2010 Mathematics Subject Classification: 60F15.

Keywords and phrases: simple linear errors-in-variables model, least square estimator, strong consistency, $\psi$-mixing random variable.

Received June 21, 2016

(ㄷ) 2016 Scientific Advances Publishers 
The most basic form of the regression models is the simple linear EV model:

$$
\eta_{i}=\theta+\beta x_{i}, \quad \xi_{i}=x_{i}+\delta_{i}, \quad 1 \leq i \leq n,
$$

with the following assumptions:

(1) $\theta, \beta, x_{i}, 1 \leq i \leq n$, are unknown constants (parameters);

(2) $\left(\varepsilon_{i}, \delta_{i}\right), 1 \leq i \leq n$, are random vectors;

(3) $\eta_{i}, \xi_{i}, 1 \leq i \leq n$, are observable.

By (1.1), we have

$$
\eta_{i}=\theta+\beta \xi_{i}+\left(\varepsilon_{i}-\beta \delta_{i}\right), \quad 1 \leq i \leq n .
$$

Then considering (1.2) as an ordinary linear regression model of $\eta_{i}$ on $\xi_{i}$ with the errors $\varepsilon_{i}-\beta \delta_{i}$, we get the least square (LS) estimators $\hat{\beta}_{n}$ and $\hat{\theta}_{n}$ of the unknown parameters $\beta$ and $\theta$ as

$$
\hat{\beta}_{n}=\frac{\sum_{i=1}^{n}\left(\xi_{i}-\bar{\xi}_{n}\right)\left(\eta_{i}-\bar{\eta}_{n}\right)}{\sum_{i=1}^{n}\left(\xi_{1}-\bar{\xi}_{n}\right)^{2}}, \quad \hat{\theta}_{n}=\bar{\eta}_{n}-\hat{\beta}_{n} \bar{\xi}_{n}
$$

where $\bar{\xi}_{n}=n^{-1} \sum_{i=1}^{n} \xi_{i}$ and $\bar{\eta}_{n}=n^{-1} \sum_{i=1}^{n} \eta_{i}$.

Deaton [1] first added the measurement errors to the usual regression model and proposed the concept of EV model. The change makes EV model more practical than the usual model. Fuller [2] summarized the early results about measurement errors in linear EV model. Mittag [3] studied the estimations of the unknown parameters in simple EV model. Gao and Liang [4] proved the strong consistency and asymptotic normality of generalized least square estimators in linear EV model. The strong and weak consistency estimators of the parameters in varying-coefficients structural linear EV model were proved by Ouyang [5]. Liu and Chen [6] proved that the weak and strong consistency are equivalent. Miao et al. [7] generalized the results of Liu and Chen [6] and obtained the rate of the strong consistency. 
All of the above results are under the condition that errors are independent identically distributed random variables, and this condition is very difficult to meet in the real world. So, there are still a lot of restrictions on practicality. In recent years, many authors put their eyes into weakening the assumption of independence. Fazekas and Kukush [8] started to add $\alpha$-mixing errors into the nonlinear EV model, however, they considered that the LS estimators of the unknown parameters were not consistency, and just proved the consistency of another alternative estimators. Also under the assumption of $\alpha$-mixing errors, the consistency of the estimators which were based on the Fourier transform in EV model was studied by Baran [9], without considering the LS estimation. Fan et al. [10] gave the strong consistency of the LS estimators under the condition that the errors in variables are stationary $\alpha$-mixing random variables, but he did not show the convergence rates. Miao et al. [11] assumed that the errors in variables $\left\{\left(\varepsilon_{n}, \delta_{n}\right), n \geq 1\right\}$ are negatively dependent random sequences, and proved the strong consistency and asymptotic normality of the LS estimators of $\beta$ and $\theta$.

In the paper, under the assumptions that the errors in variables are $\psi$-mixing, we not only prove the strong consistency of the LS estimators $\hat{\beta}_{n}$ and $\hat{\theta}_{n}$ of the unknown parameters $\beta$ and $\theta$, but also obtain the convergence rates of $\hat{\beta}_{n}-\beta$ and $\hat{\theta}_{n}-\theta$. Although a sequence of $\psi$-mixing random variables is also $\alpha$-mixing (see $\mathrm{Lu}$ and $\mathrm{Lin}[12]$ ), but we have more limit properties under $\psi$-mixing setup and hence we can obtain more exact results in EV model.

We firstly introduce the concept of the $\psi$-mixing random variables (or random vectors). 
Definition 1.1. Define the $\psi$-mixing coefficient for $\left\{X_{n}, n \geq 1\right\}$, a sequence of random variables or random vectors, as

$$
\psi(n)=\sup _{m \geq 1} \sup _{A \in \mathcal{F}_{1}^{m}, B \in \mathcal{F}_{m+n}^{\infty} P(A) P(B) \neq 0}\left|\frac{P(A B)}{P(A) P(B)}-1\right|,
$$

where $\mathcal{F}_{n}^{m}=\sigma\left(X_{i}: n \leq i \leq m\right)$. Then $\left\{X_{n}, n \geq 1\right\}$ is said to be $\psi$-mixing, if $\psi(n) \rightarrow 0$ as $n \rightarrow \infty$.

The concept of $\psi$-mixing is introduced by Blum et al. [13]. For more detail, we refer to the book of $\mathrm{Lu}$ and Lin [12].

We now state the main results. Some lemmas and the proofs of the main results will be detailed in the next section.

Theorem 1.1. Under model (1.1), suppose that $\left\{\left(\varepsilon_{n}, \delta_{n}\right), n \geq 1\right\}$ is a sequence of identically distributed $\psi$-mixing random vectors with $\sum_{n=1}^{\infty} \psi(n)<\infty$. If $E \varepsilon_{1}=E \delta_{1}=0, E\left|\varepsilon_{1}\right|^{p}<\infty, E\left|\delta_{1}\right|^{p}<\infty$, and

$$
\lim _{n \rightarrow \infty} \frac{S_{n}}{n^{2-2 / p}}=\infty
$$

for some $p \geq 2$, where $S_{n}=\sum_{i=1}^{n}\left(x_{i}-\bar{x}_{n}\right)^{2}$ and $\bar{x}_{n}=n^{-1} \sum_{i=1}^{n} x_{i}$. Then

$$
\frac{\sqrt{S_{n}}}{n^{1 / p}}\left(\hat{\beta}_{n}-\beta\right) \rightarrow 0 \text { a.s. }
$$

Remark 1.1. It is easy to show that $\sqrt{S_{n}} / n^{1 / p} \rightarrow \infty$ by (1.4) since $p \geq 2$, thus $\hat{\beta}_{n} \rightarrow \beta$ almost surely by (1.5). So, we not only obtain the strong consistency estimators of $\beta$, but also obtain the convergence rate.

Theorem 1.2. Under the conditions of Theorem 1.1, further assume that

$$
\limsup _{n \rightarrow \infty} \frac{n^{1-\alpha+1 / p}}{\sqrt{S_{n}}} \cdot\left|\bar{x}_{n}\right|<\infty
$$

for some $\alpha \in(1 / 2,1)$. Then 


$$
n^{1-\alpha}\left(\hat{\theta}_{n}-\theta\right) \rightarrow 0 \text { a.s. }
$$

\section{Lemmas and Proofs of Main Results}

To prove the main results, we need the following two lemmas. The first one is from Lu and Lin [12] and the second one is due to Yang [14].

Lemma 2.1. Let $\left\{X_{n}, n \geq 1\right\}$ be a sequence of identically distributed $\psi$-mixing random variables with $\sum_{n=1}^{\infty} \psi\left(2^{n}\right)<\infty$. Suppose that $E\left|X_{1}\right|^{r}<\infty$ for some $1 \leq r<2$, then

$$
n^{-1} \sum_{i=1}^{n}\left(X_{i}-E X_{i}\right)=o\left(n^{-(1-1 / r)}\right) \text { a.s. }
$$

Lemma 2.2. Let $p \geq 2,\left\{X_{n}, n \geq 1\right\}$ be a sequence of identically distributed $\psi$-mixing random variables with $\sum_{n=1}^{\infty} \psi(n)<\infty,\left\{a_{n i}, n \geq 1,1 \leq i \leq n\right\}$ an array of constants with $\sup _{n \geq 1} \max _{1 \leq i \leq n}\left|a_{n i}\right|<\infty$ and

$$
\limsup _{n \rightarrow \infty} n^{-\theta} \sum_{i=1}^{n}\left|a_{n i}\right|^{2}<\infty
$$

for some $\theta \in(0,2 / p)$. Then $E X_{1}=0$ and $E\left|X_{1}\right|^{p}<\infty$ imply that

$$
n^{-1 / p} \sum_{i=1}^{n} a_{n i} X_{i} \rightarrow 0 \text { a.s. }
$$

Proof of Theorem 1.1. By (1.1) and (1.3), it is easy to show that

$$
\begin{aligned}
& \hat{\beta}_{n}-\beta \\
& =\frac{\sum_{i=1}^{n}\left(\xi_{i}-\bar{\xi}_{n}\right)\left(\eta_{i}-\bar{\eta}_{n}\right)-\beta \sum_{i=1}^{n}\left(\xi_{i}-\bar{\xi}_{n}\right)^{2}}{\sum_{i=1}^{n}\left(\xi_{i}-\bar{\xi}_{n}\right)^{2}} \\
& =\frac{\sum_{i=1}^{n}\left[\left(x_{i}-\bar{x}_{n}\right)+\left(\delta_{i}-\bar{\delta}_{n}\right)\right] \cdot\left[\beta\left(x_{i}-\bar{x}_{n}\right)+\left(\varepsilon_{i}-\bar{\varepsilon}_{n}\right)\right]-\beta \sum_{i=1}^{n}\left[\left(x_{i}-\bar{x}_{n}\right)+\left(\delta_{i}-\bar{\delta}_{n}\right)\right]^{2}}{\sum_{i=1}^{n}\left(\xi_{i}-\bar{\xi}_{n}\right)^{2}}
\end{aligned}
$$




$$
=\frac{\sum_{i=1}^{n}\left(\delta_{i}-\bar{\delta}_{n}\right) \varepsilon_{i}+\sum_{i=1}^{n}\left(x_{i}-\bar{x}_{n}\right)\left(\varepsilon_{i}-\beta \delta_{i}\right)-\beta \sum_{i=1}^{n}\left(\delta_{i}-\bar{\delta}_{n}\right)^{2}}{\sum_{i=1}^{n}\left(\xi_{i}-\bar{\xi}_{n}\right)^{2}} .
$$

Hence to prove (1.5), it suffices to prove that

$$
\begin{aligned}
& \frac{1}{\sqrt{S_{n}} n^{1 / p}} \sum_{i=1}^{n}\left(\delta_{i}-\bar{\delta}_{n}\right) \varepsilon_{i} \rightarrow 0 \text { a.s., } \\
& \frac{1}{\sqrt{S_{n}} n^{1 / p}} \sum_{i=1}^{n}\left(x_{i}-\bar{x}_{n}\right)\left(\varepsilon_{i}-\beta \delta_{i}\right) \rightarrow 0 \text { a.s., } \\
& \frac{1}{\sqrt{S_{n}} n^{1 / p}} \sum_{i=1}^{n}\left(\delta_{i}-\bar{\delta}_{n}\right)^{2} \rightarrow 0 \text { a.s., } \\
& \frac{1}{S_{n}} \sum_{i=1}^{n}\left(\xi_{i}-\bar{\xi}_{n}\right)^{2} \rightarrow 1 \text { a.s. }
\end{aligned}
$$

By Lemma 2.1,

$$
n^{-1} \sum_{i=1}^{n} \delta_{i}^{2} \rightarrow E \delta_{1}^{2} \quad \text { a.s., and } n^{-1} \sum_{i=1}^{n} \varepsilon_{i}^{2} \rightarrow E \varepsilon_{1}^{2} \text { a.s. }
$$

Thus by (2.4) and (2.9),

$$
\frac{1}{\sqrt{S_{n}} n^{1 / p}} \sum_{i=1}^{n}\left(\delta_{i}-\bar{\delta}_{n}\right)^{2} \leq \frac{1}{\sqrt{S_{n}} n^{1 / p}} \sum_{i=1}^{n} \delta_{i}^{2}=\frac{n^{1-1 / p}}{\sqrt{S_{n}}} \cdot n^{-1} \sum_{i=1}^{n} \delta_{i}^{2} \rightarrow 0 \text { a.s., }
$$

and

$$
\frac{1}{\sqrt{S_{n}} n^{1 / p}} \sum_{i=1}^{n} \varepsilon_{i}^{2}=\frac{n^{1-1 / p}}{\sqrt{S_{n}}} \cdot n^{-1} \sum_{i=1}^{n} \varepsilon_{i}^{2} \rightarrow 0 \text { a.s. }
$$

Then

$$
\frac{1}{\sqrt{S_{n}} n^{1 / p}}\left|\sum_{i=1}^{n}\left(\delta_{i}-\bar{\delta}_{n}\right) \varepsilon_{i}\right| \leq \frac{1}{2 \sqrt{S_{n}} n^{1 / p}} \sum_{i=1}^{n}\left[\left(\delta_{i}-\bar{\delta}_{n}\right)^{2}+\varepsilon_{i}^{2}\right] \rightarrow 0 \text { a.s. }
$$


So (2.5) and (2.7) hold. Set $a_{n i}=\left(x_{i}-\bar{x}_{n}\right) / \sqrt{S_{n}}, 1 \leq i \leq n, n \geq 1$. Then $\sum_{i=1}^{n} a_{n i}^{2}=1$ for all $n \geq 1$ and by Lemma 2.2 ,

$$
\frac{1}{n^{1 / p}} \sum_{i=1}^{n} a_{n i}\left(\varepsilon_{i}-\beta \delta_{i}\right) \rightarrow 0 \text { a.s. }
$$

which follows (2.6). We finally prove (2.8). Note that

$$
\sum_{i=1}^{n}\left(\xi_{i}-\bar{\xi}_{n}\right)^{2}=\sum_{i=1}^{n}\left(x_{i}-\bar{x}_{n}\right)^{2}+2 \sum_{i=1}^{n}\left(x_{i}-\bar{x}_{n}\right)\left(\delta_{i}-\bar{\delta}_{n}\right)+\sum_{i=1}^{n}\left(\delta_{i}-\bar{\delta}_{n}\right)^{2} .
$$

Then by the Hölder's inequality,

$$
\begin{aligned}
& \left|\frac{1}{S_{n}} \sum_{i=1}^{n}\left(\xi_{i}-\bar{\xi}_{n}\right)^{2}-1\right| \\
& \quad=\frac{1}{S_{n}}\left|2 \sum_{i=1}^{n}\left(x_{i}-\bar{x}_{n}\right)\left(\delta_{i}-\bar{\delta}_{n}\right)+\sum_{i=1}^{n}\left(\delta_{i}-\bar{\delta}_{n}\right)^{2}\right| \\
& \quad \leq \frac{1}{S_{n}}\left(2 \sum_{i=1}^{n}\left|\left(x_{i}-\bar{x}_{n}\right)\left(\delta_{i}-\bar{\delta}_{n}\right)\right|+\sum_{i=1}^{n}\left(\delta_{i}-\bar{\delta}_{n}\right)^{2}\right) \\
& \quad \leq \frac{1}{S_{n}}\left(2 \sqrt{\sum_{i=1}^{n}\left(x_{i}-\bar{x}_{n}\right)^{2}} \sqrt{\sum_{i=1}^{n}\left(\delta_{i}-\bar{\delta}_{n}\right)^{2}}+\sum_{i=1}^{n}\left(\delta_{i}-\bar{\delta}_{n}\right)^{2}\right) \\
& \quad=2 \sqrt{\frac{1}{S_{n}} \sum_{i=1}^{n}\left(\delta_{i}-\bar{\delta}_{n}\right)^{2}}+\frac{1}{S_{n}} \sum_{i=1}^{n}\left(\delta_{i}-\bar{\delta}_{n}\right)^{2} .
\end{aligned}
$$

By (2.4) and (2.5),

$$
\frac{1}{S_{n}} \sum_{i=1}^{n}\left(\delta_{i}-\bar{\delta}_{n}\right)^{2}=\frac{1}{\sqrt{S_{n}} n^{1 / p}} \sum_{i=1}^{n}\left(\delta_{i}-\bar{\delta}_{n}\right)^{2} \cdot \frac{n^{1-1 / p}}{\sqrt{S_{n}}} \cdot n^{2 / p-1} \rightarrow 0 \text { a.s. }
$$


which by (2.10) ensures that

$$
\frac{1}{S_{n}} \sum_{i=1}^{n}\left(\xi_{i}-\bar{\xi}_{n}\right)^{2} \rightarrow 1 \text { a.s. }
$$

i.e., (2.8) holds. The proof is completed by (2.5), (2.6), (2.7), and (2.8).

Proof of Theorem 1.2. By (1.3), we have

$$
\hat{\theta}_{n}-\theta=\left(\beta-\hat{\beta}_{n}\right) \bar{x}_{n}+\left(\beta-\hat{\beta}_{n}\right) \bar{\delta}_{n}-\beta \bar{\delta}_{n}+\bar{\varepsilon}_{n} .
$$

By $E\left|\varepsilon_{1}\right|^{p}<\infty, E\left|\delta_{1}\right|^{p}<\infty$ and Lemma 2.1, we have for any $\alpha \in(1 / 2,1)$

$$
n^{1-\alpha} \bar{\varepsilon}_{n} \rightarrow 0 \text { a.s., } \quad n^{1-\alpha} \bar{\delta}_{n} \rightarrow 0 \text { a.s. }
$$

By Theorem 1.1, $\hat{\beta}_{n} \rightarrow \beta$ almost surely and hence by (2.12),

$$
n^{1-\alpha}\left(\beta-\hat{\beta}_{n}\right) \bar{\delta}_{n} \rightarrow 0 \text { a.s. }
$$

By Theorem 1.1 and (1.6), we have

$$
n^{1-\alpha}\left(\beta-\hat{\beta}_{n}\right) \bar{x}_{n}=\frac{n^{1-\alpha+1 / p}}{\sqrt{S_{n}}} \bar{x}_{n} \cdot \frac{\sqrt{S_{n}}}{n^{1 / p}}\left(\beta-\hat{\beta}_{n}\right) \rightarrow 0 \text { a.s. }
$$

Therefore, by (2.11), (2.12), (2.13), and (2.14),

$$
n^{1-\alpha}\left(\hat{\theta}_{n}-\theta\right)=n^{1-\alpha}\left[\left(\beta-\hat{\beta}_{n}\right) \bar{x}_{n}+\left(\beta-\hat{\beta}_{n}\right) \bar{\delta}_{n}-\beta \bar{\delta}_{n}+\bar{\varepsilon}_{n}\right] \rightarrow 0 \text { a.s., }
$$

i.e., (1.7) holds. The proof is completed.

\section{Acknowledgements}

The research is supported by the National Natural Science Foundation of China (No. 11271161). 


\section{References}

[1] A. Deaton, Panel data from time series of cross-sections, J. Econ. 30(1) (1985), 109-126.

[2] W. A. Fuller, Measurement Error Models, New York, 1987.

[3] H. J. Mittag, Estimating parameters in a simple errors-in-variables model: A new approach base on finite sample distribution theory, Stat. Papers 30(1) (1989), 133-140.

[4] Y. F. Gao and H. Liang, On generalized least square estimates in EV linear regression model, Mathematics in Practice and Theory (4) (1996), 343-348 (in Chinese).

[5] G. Ouyang, On parameter estimation for linear varying-coefficients structural EV models, Acta Math. Appl. Sinica (1) (2005), 73-85 (in Chinese).

[6] J. X. Liu and X. R. Chen, Consistency of LS estimator in simple linear EV regression models, Acta Math. Sci. Ser. B, Engl. Ed. 25(1) (2005), 50-58.

[7] Y. Miao, K. Wang and F. F. Zhao, Some limit behaviors for the LS estimator in simple linear EV regression models, Statist. Probab. Letters 81(1) (2011), 92-102.

[8] I. Fazekas and A. G. Kukush, Asymptotic properties of an estimator in nonlinear functional errors-in-variables models with dependent error terms, Comp. Math. Appl. 34(10) (1997), 23-39.

[9] S. Baran, A consistent estimator for linear models with dependent observations, Comm. Statis.: Theory Methods 33(10) (2004), 2469-2486.

[10] G. L. Fan, H. Y. Liang, J. F. Wang et al., Asymptotic properties for LS estimators in EV regression model with dependent errors, Asta Advances in Statistical Analysis 94(1) (2010), 89-103.

[11] Y. Miao, F. F. Zhao, K. Wang et al., Asymptotic normality and strong consistency of LS estimators in the EV regression model with NA errors, Stat. Papers 54(1) (2013), 193-206.

[12] C. Y. Lu and Z. Y. Lin, Limit Theory for Mixing Random Variables, Dordrecht/Beijing: Kluwer Academic Publishers/Science Press, 1997.

[13] J. R. Blum, D. L. Hanson and L. Koopmans, On the strong law of large numbers for a class of stochastic processes, Z. Wahrsch. Verw. Gebiete 2 (1963), 1-11.

[14] S. C. Yang, Almost sure convergence of weighted sums of mixing sequences, J. Sys. Sci. Math. Scis. 15(3) (1995), 254-265. 